\title{
PID controller adjustment for MA-LFC by using imperialist competitive algorithm
}

\begin{abstract}
In this paper a new evolutionary computing method based on Imperialist Competitive Algorithm (ICA) is used for tuning the elements of a PID controller which is applied in a Multi Area Load Frequency Control System (MA-LFC). If a large power imbalance is suddenly happened in a multi area power electric system, generation units and also consumer sides will be affected by the distortion in the energy balance between both two sides. This inequality is firstly handled by the kinetic energy of the system turning components, but, eventually, the frequency will change. Therefore, LFC is considered as one of the most challenging issues in power system control and operation. PID type controllers are conventional solutions for MA-LFC. The three parameters of the PID controllers have been adjusted traditionally. In this paper, a PID controller is applied for the MA-LFC problem and then its parts are modified by using ICA method. To validate the application of the technique, a multi area network with some uncertainties is provided. Finally the results of the ICAPID controller are compared with the ones of GA optimized controllers. The simulation results show the success and the validity of the ICA-PID controller in compare with the GA - PID controller.
\end{abstract}

Keyword: Genetic algorithm; Imperialist competitive algorithm; Load frequency control; Multi area network; Power system dynamics 